\title{
ON THE VORONOI REGIONS OF CERTAIN LATTICES*
}

\author{
J. H. CONWAY† AND N. J. A. SLOANE $\ddagger$
}

\begin{abstract}
The Voronoi region of a lattice $L_{n} \subseteq \mathbb{R}^{n}$ is the convex polytope consisting of all points of $\mathbb{R}^{n}$ that are closer to the origin than to any other point of $L_{n}$. In this paper we calculate the second moments of the Voronoi regions of the lattices $E_{6}^{*}, E_{7}^{*}, K_{12}, \Lambda_{16}$ and $\Lambda_{24}$. The results show that these lattices are the best quantizers presently known in dimensions $6,7,12,16$ and 24 . The calculations are performed by Monte Carlo integration, and make use of fast algorithms for finding the closest lattice point to an arbitrary point of the space. We also establish two general theorems concerning the number of faces of the Voronoi region of a lattice.
\end{abstract}

AMS(MOS) subject classifications. Primary, 10E05, 52A45

1. Introduction. The Voronoi region of an $n$-dimensional lattice $L_{n} \subseteq \mathbb{R}^{n}$ is the convex polytope

$$
\mathscr{P}:=\left\{x \in \mathbb{R}^{n}: N(x) \leqq N(x-l) \text { for all } l \in L_{n}\right\},
$$

where $N(x)=x \cdot x$ denotes the norm of a vector (cf. [10] and the references given there). Figure 1 for example shows the Voronoi region of the body-centered cubic lattice $A_{3}^{*}$. If $L_{n}$ is used as a quantizer (or analog-to-digital convertor), its average mean squared error per symbol is given by

$$
G\left(L_{n}\right):=\frac{1}{n} \frac{\int_{\mathscr{P}} x \cdot x d x}{\operatorname{det}\left(L_{n}\right)^{(n+2) /(2 n)}},
$$

where det $\left(L_{n}\right)$, the determinant of $L_{n}$, is the square of the volume of $\mathscr{P}$ ([10], [22], [39]). $G\left(L_{n}\right)$ is a normalized second moment of $\mathscr{P}$ about the origin. (The formula (1) assumes that the input to the quantizer is uniformly distributed over a large region of $\mathbb{R}^{n}$, and the number of output levels is very large.)

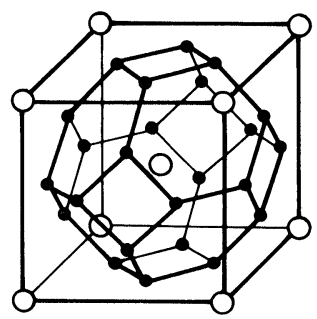

FIG. 1. The Voronoi region of the body-centered cubic lattice $A_{3}^{*}\left(\cong D_{3}^{*}\right)$ is a truncated octahedron. The open circles represent lattice points, the solid circles the vertices of the Voronoi region.

$G\left(L_{n}\right)$ measures the average error introduced when points of $\mathbb{R}^{n}$ are replaced by the closest lattice points. If we use the 1 -dimensional integer lattice $\mathbb{Z}$ as a quantizer, the average error is $G(\mathbb{Z})=1 / 12=0.08333 \cdots$. But by using higher-dimensional lattices this can be reduced (see Table 1 and Fig. 2). The expression for $G\left(L_{n}\right)$ in (1) has been scaled so as to provide a proper comparison between quantizers of different dimensions.

* Received by the editors February 7, 1983, and in revised form May 27, 1983.

$\dagger$ Department of Pure Mathematics and Mathematical Statistics, University of Cambridge, Cambridge CB2 1SB, England.

$\ddagger$ Mathematics and Statistics Research Center, Bell Laboratories, Murray Hill, New Jersey 07974. 
TABLE 1

Smallest mean squared error $G\left(L_{n}\right)$ of any known $n$-dimensional lattice $L_{n}$

\begin{tabular}{ccl}
\hline $\begin{array}{c}\text { dimension } \\
n\end{array}$ & $\begin{array}{c}\text { lattice } \\
L_{n}\end{array}$ & \multicolumn{1}{c}{ mean squared error } \\
$G\left(L_{n}\right)$
\end{tabular}

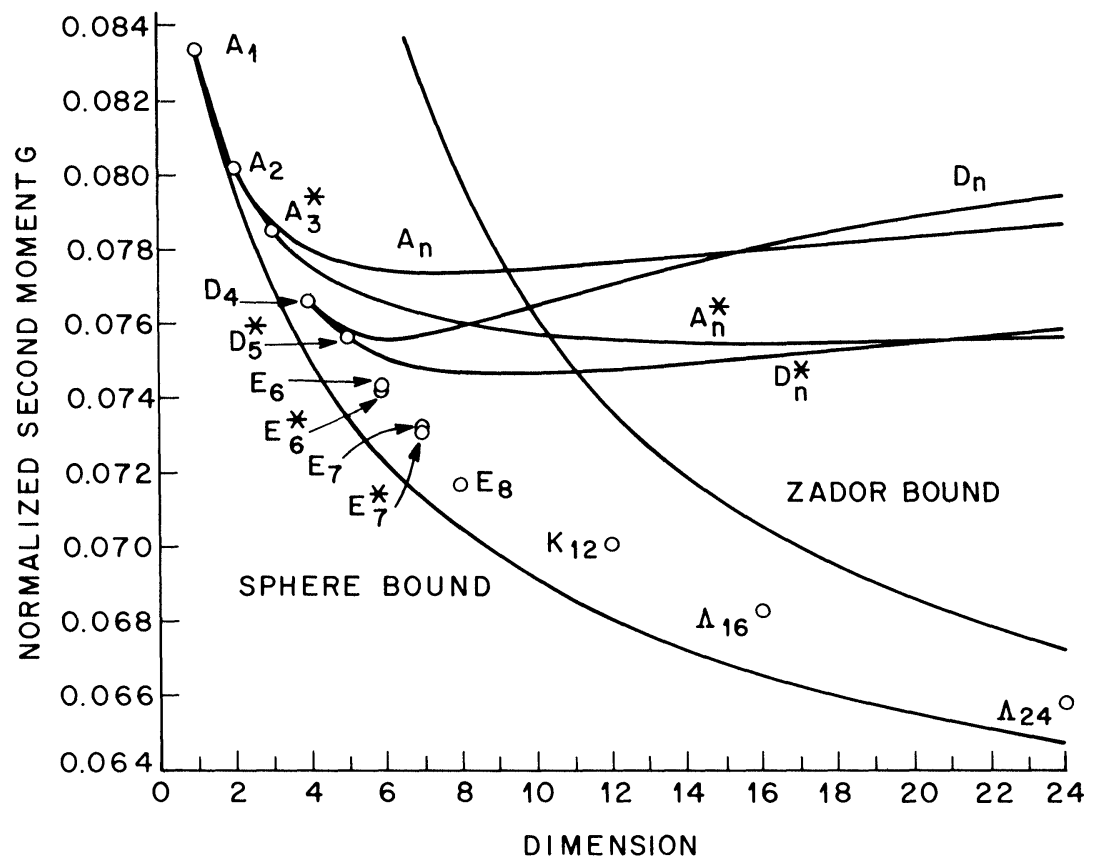

FIG. 2. Normalized second moment $G$ for various lattices, and the Zador and sphere bounds. It is known that the best quantizers must lie between the two bounds.

In 1953 Fejes Tóth ([20], see also [30]) proved that the hexagonal lattice $A_{2}$ is the optimal lattice quantizer in two dimensions, i.e., has the smallest value of $G\left(L_{2}\right)$, namely $5 / 36 \sqrt{3}=0.0801875 \cdots$. He also showed that no nonlattice quantizer can do better. Gersho [22] computed $G\left(A_{3}\right), G\left(A_{3}^{*}\right)$ and $G\left(D_{4}\right)$, and conjectured that $A_{3}^{*}\left(\cong D_{3}^{*}\right)$ is the optimal lattice in three dimensions. This conjecture was established in [2]. In an earlier paper [10] we determined the Voronoi regions and evaluated $G$ for all the root lattices $A_{n}(n \geqq 1), D_{n}(n \geqq 3), E_{6}, E_{7}, E_{8}\left(=E_{8}^{*}\right)$ and the dual lattices $A_{n}^{*}(n \geqq 1)$ and $D_{n}^{*}(n \geqq 3)$. We observed that the optimal lattice quantizer was often 
the dual of the densest lattice packing, and conjectured that this may be true in general. It is true in dimensions 1, 2 and 3, and is supported by the available data in dimensions 4, 5 and 8. In the present paper we evaluate $G$ for the duals of the densest known packings in $6,7,12,16$ and 24 dimensions, namely for the lattices $E_{6}^{*}, E_{7}^{*}$, the Coxeter-Todd lattice $K_{12}\left(\cong K_{12}^{*}\right)$, the Barnes-Wall lattice $\Lambda_{16}\left(\cong \Lambda_{16}^{*}\right)$, and the Leech lattice $\Lambda_{24}\left(=\Lambda_{24}^{*}\right)$. The results are summarized in Table 1 and Fig. 2 and support our conjecture.

It is worth pointing out that this conjecture would imply the somewhat surprising result that the best quantizer is in general different from the most efficient lattice covering of space by spheres. Indeed, the two problems already have different answers in dimensions 4 and 5 . There the best lattice coverings are known to be $A_{4}^{*}$ and $A_{5}^{*}$ [33], yet $D_{4}^{*}$ and $D_{5}^{*}$ are better quantizers (see Fig. 2). In higher dimensions, the best coverings known are $A_{n}^{*}$, if $n \leqq 23$, and then various lattices constructed from $\Lambda_{24}[1]$. So it seems likely that, in all dimensions between 4 and 23 , the best lattice quantizers and coverings are distinct.

The values of $G$ for various lattices are compared in Fig. 2, the values for $A_{n}$, $A_{n}^{*}, D_{n}, D_{n}^{*}, E_{6}, E_{7}$ and $E_{8}$ being taken from [10]. In 1964 Zador proved by a nonconstructive argument that good quantizers exist in sufficiently high dimensions, and observed that the second moment of a sphere gives a bound in the other direction (see [39], and also [22], [10, Eq. (3)]). These two bounds are also plotted in the figure.

We use Monte Carlo integration to compute $G$. The technique is briefly described in $\S 2$. It requires that we have a fast algorithm for performing the quantizing, that is, given an arbitrary point of $\mathbb{R}^{n}$, for finding the closest lattice point. (If the lattice is used as a code for a band-limited channel, this algorithm performs the decoding [9], [11].) The best quantizing algorithms we have found for these lattices, and the values of $G$ that were obtained, are given in $\$ \S 3-7$.

If a more complete description of the polytopes $\mathscr{P}$ were available, we could determine $G$ exactly by decomposing each polytope into simplices and using the methods of [10]. Unfortunately little is known about the polytopes of these lattices. For $E_{6}^{*}$ and $E_{7}^{*}$, for example, even the covering radius (the distance of the furthest vertex of $\mathscr{P}$ from the origin) is unknown, although it is known for all the other lattices mentioned [8], [10], [13], [15], [17]. In $\S 8$ we establish two theorems which help determine the number of $(n-1)$-dimensional faces of $\mathscr{P}$, and in the last section we use them to study the Voronoi region of $K_{12}$.

Notation. If $L_{n}$ is a lattice in $\mathbb{R}^{n}$ (the subscript indicates the dimension), the dual lattice $L_{n}^{*}=\left\{x \in \mathbb{R}^{n}: x \cdot y \in \mathbb{Z}\right.$ for all $\left.y \in L_{n}\right\}$. Two lattices $L_{n}$ and $M_{n}$ are equivalent, written $L_{n} \cong M_{n}$, if they differ only by a rotation and possibly a change of scale. The direct sum of $k$ copies of $L_{n}$ is written $L_{n}^{k}$. For further information about these lattices see [4], [10], [13]-[17], [27], [31], [36], [37].

2. Monte Carlo integration over a Voronoi region. The Monte Carlo technique that we use to calculate $G$ is slightly unusual. We wish to find $I:=\int_{\mathscr{P}} x \cdot x d x$. Conventional Monte Carlo methods ([24], [25], [28], [34]) would begin by replacing $I$ by $\int_{\mathscr{Q}} \chi_{\mathscr{P}}(x) x \cdot x d x$, where 2 is a region enclosing $\mathscr{P}$, usually a sphere or a cube, and $\chi_{\mathscr{P}}(x)$ is 1 if $x \in \mathscr{P}, 0$ otherwise. This is wasteful, since points in $\mathscr{Q} \backslash \mathscr{P}$ do not contribute to the estimate. The following approach avoids this difficulty, by exploiting our fast quantizing algorithms.

Let $v^{(1)}, \cdots, v^{(n)}$ be linearly independent vectors spanning the lattice, and let $u_{1}, \cdots, u_{n}$ be independent random numbers, uniformly distributed between 0 and 1 . Then $y=\Sigma u_{i} v^{(i)}$ is uniformly distributed over the fundamental parallelepiped generated 
by the $v^{(i)}$. Let $l$ be the closest lattice point to $y$ (found by the quantizing algorithm for this lattice); then $w(y):=y-l$ is uniformly distributed over the Voronoi region $\mathscr{P}$. Figure 3 illustrates this for the hexagonal lattice $A_{2}$.

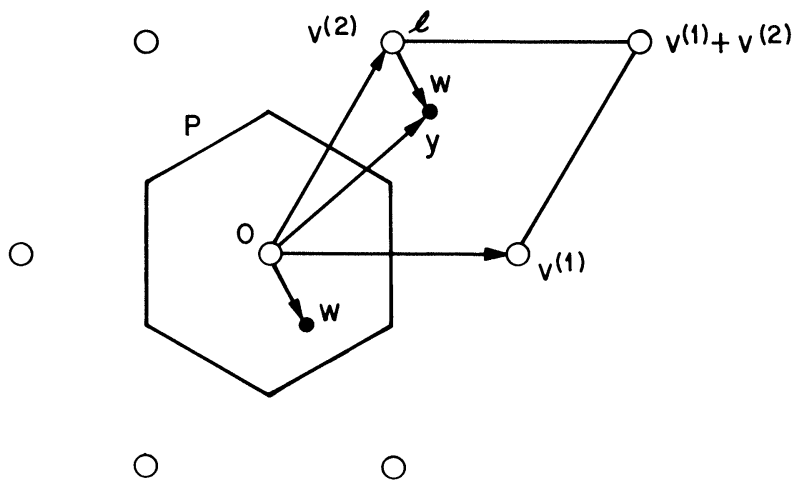

FIG. 3. Hexagonal lattice $A_{2}$ with spanning vectors $v^{(1)}, v^{(2)} ; y$ is a random point in the parallelogram $0, v^{(1)}, v^{(2)}, v^{(1)}+v^{(2)} ; l$ is the closest lattice point to $y$; and $w=y-l$ is a random point in the Voronoi region $\mathscr{P}$.

Then if $m=g h$ random points $y^{(0)}, \cdots, y^{(m-1)}$ are selected in the manner just described,

$$
\hat{I}=\frac{1}{m} \sum_{i=0}^{m-1} N\left(w\left(y^{(i)}\right)\right)
$$

is an estimate of $I$, and when suitably scaled (see (1)) produces our estimate $\hat{G}$ of $G$. To estimate the variance of $\hat{I}$, we group the measurements into $g$ sets of $h$, and use the jackknife estimator (see [29], [32], [38]):

$$
\widehat{\operatorname{var}(\hat{I})}=\frac{1}{g(g-1)} \sum_{j=0}^{g-1}\left(\frac{1}{h} A_{j}-\hat{I}\right)^{2}
$$

where

$$
A_{j}=\sum_{i=j h}^{(j+1) h-1} N\left(w\left(y^{(i)}\right)\right), \quad j=0,1, \cdots, h-1 .
$$

By taking the square root of (3) and scaling, we obtain an estimate $\hat{\sigma}$ for the standard deviation of $\hat{G}$, and then $\hat{G} \pm 2 \hat{\sigma}$ is our final estimate for $G$. The portable random number generator on the PORT library [21], which combines a congruential generator and a Tausworthe generator, was used to produce the $u_{i}$ 's.

To test this procedure we applied it first to the lattices $E_{6}, E_{7}$ and $E_{8}$, for which the exact value of $G$ is known [10]. The estimates agreed closely with the exact values. For example, for $E_{8}$ we found using $m=10^{7}$ points ( $g$ was always taken to be 100 ) that

$$
\hat{G}\left(E_{8}\right)=0.071689 \pm 0.000008,
$$

while the true value is

$$
G\left(E_{8}\right)=\frac{929}{12960}=0.0716821 \cdots
$$


3. The lattices $\boldsymbol{E}_{6}$ and $\boldsymbol{E}_{6}^{*} . E_{6}$ is most easily obtained from the complex 3dimensional $\mathbb{Z}[\omega]$-lattice with generator matrix

$$
\left[\begin{array}{lll}
\theta & 0 & 0 \\
0 & \theta & 0 \\
1 & 1 & 1
\end{array}\right], \quad \omega=e^{2 \pi i / 3}, \quad \theta=\omega-\bar{\omega}=i \sqrt{3},
$$

([16, p. 421], [35, Example 5], [36, § 5.8.2], [37, § 17]), and the dual lattice $E_{6}^{*}$ from

$$
\left[\begin{array}{rrr}
\theta & 0 & 0 \\
1 & -1 & 0 \\
1 & 0 & -1
\end{array}\right]
$$

Thus the real 6-dimensional lattices $E_{6}$ and $E_{6}^{*}$ have generator matrices

(7a)

(7b)

$$
\begin{aligned}
& {\left[\begin{array}{cccccc}
0 & \sqrt{3} & 0 & 0 & 0 & 0 \\
0 & 0 & 0 & \sqrt{3} & 0 & 0 \\
1 & 0 & 1 & 0 & 1 & 0 \\
\frac{-3}{2} & \frac{-\sqrt{3}}{2} & 0 & 0 & 0 & 0 \\
0 & 0 & \frac{-3}{2} & \frac{-\sqrt{3}}{2} & 0 & 0 \\
\frac{-1}{2} & \frac{\sqrt{3}}{2} & \frac{-1}{2} & \frac{\sqrt{3}}{2} & \frac{-1}{2} & \frac{\sqrt{3}}{2}
\end{array}\right],} \\
& {\left[\begin{array}{cccccc}
0 & \sqrt{3} & 0 & 0 & 0 & 0 \\
1 & 0 & -1 & 0 & 0 & 0 \\
1 & 0 & 0 & 0 & -1 & 0 \\
\frac{-3}{2} & \frac{-\sqrt{3}}{2} & 0 & 0 & 0 & 0 \\
\frac{-1}{2} & \frac{\sqrt{3}}{2} & \frac{1}{2} & \frac{-\sqrt{3}}{2} & 0 & 0 \\
\frac{-1}{2} & \frac{\sqrt{3}}{2} & 0 & 0 & \frac{1}{2} & \frac{-\sqrt{3}}{2}
\end{array}\right]}
\end{aligned}
$$

respectively. (For example (7a) is obtained from the real and imaginary parts of (5) and $\omega$ times (5).)

To find a quantizing algorithm for any of these lattices, we proceed as follows, following [11]. Inside our lattice $L_{n}$ we look for a sublattice $S_{n}$, of small index $t$ (say), for which quantizing is easy. Suppose

$$
L_{n}=\left(a^{(0)}+S_{n}\right) \cup \cdots \cup\left(a^{(t-1)}+S_{n}\right),
$$

where $a^{(0)}, \cdots, a^{(t-1)}$ are coset representatives for $S_{n}$ in $L_{n}$. Let $\phi: \mathbb{R}^{n} \rightarrow S_{n}$ be a quantizer for $S_{n}$, so that $\phi(x)$ is the closest point of $S_{n}$ to a given vector $x$. Then a quantizer for $L_{n}$ is obtained by taking the given vector $w$, forming the $t$ candidates

$$
c^{(i)}=\phi\left(w-a^{(i)}\right)+a^{(i)}, \quad i=0, \cdots, t-1,
$$

and choosing the closest candidate to $w$. 
Both $E_{6}$ and $E_{6}^{*}$ contain a sublattice $S_{6}$ isomorphic to $A_{2}^{3}$, namely the real version of the lattice $\theta \mathbb{Z}[\omega]^{3}$ with generator matrix

$$
\left[\begin{array}{lll}
\theta & 0 & 0 \\
0 & \theta & 0 \\
0 & 0 & \theta
\end{array}\right] .
$$

$S_{6}$ has index 3 in $E_{6}$, with coset representatives

$$
a^{(0)}=(0,0,0,0,0,0), \quad a^{(1)}=(1,0,1,0,1,0), \quad a^{(2)}=-a^{(1)},
$$

and has index 9 in $E_{6}^{*}$, with coset representatives given in Table 2.

TABLE 2

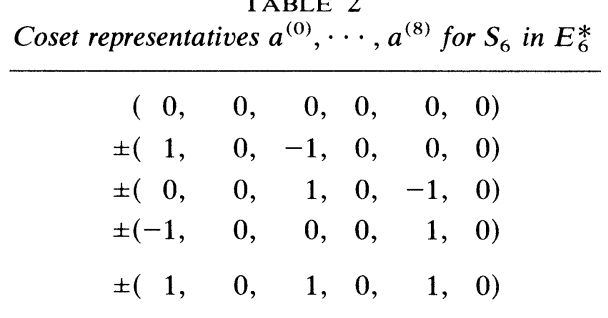

It is easy to design a quantizer for the hexagonal lattice $A_{2}$ (and hence $A_{2}^{3}$ ), either using the fact that $A_{2}$ is the union of a rectangular lattice and a translate, as suggested by Gersho [23, p. 165], or via 3-dimensional coordinates as we suggest in [10, § VII]. Gersho's method seems slightly simpler and is the one we adopted. From the previous discussion we have the following quantizing algorithms for $E_{6}$ and $E_{6}^{*}$.

QuAntizing Algorithms for $E_{6}(t=3)$ And $E_{6}^{*}(t=9)$

Given $w=\left(w_{1}, \cdots, w_{6}\right)$, to find the closest lattice point of $E_{6}\left(\right.$ or $\left.E_{6}^{*}\right)$.

Subtract one of $t$ coset representatives $a^{(i)}$, obtaining

$$
z=w-a^{(i)}=\left(z_{1}, \cdots, z_{6}\right) .
$$

Divide the corresponding complex vector by $\theta$, i.e., form

$$
z^{\prime}=\left(\frac{z_{2}}{\sqrt{3}}, \frac{-z_{1}}{\sqrt{3}}, \frac{z_{4}}{\sqrt{3}}, \frac{-z_{3}}{\sqrt{3}}, \frac{z_{6}}{\sqrt{3}}, \frac{-z_{5}}{\sqrt{3}}\right) \text {. }
$$

Apply the quantizer for $A_{2}$ to the three pairs

$$
\left(\frac{z_{2}}{\sqrt{3}}, \frac{-z_{1}}{\sqrt{3}}\right),\left(\frac{z_{4}}{\sqrt{3}}, \frac{-z_{3}}{\sqrt{3}}\right),\left(\frac{z_{6}}{\sqrt{3}}, \frac{-z_{5}}{\sqrt{3}}\right)
$$

obtaining say

$$
\left(m_{1}, m_{2}\right),\left(m_{3}, m_{4}\right),\left(m_{5}, m_{6}\right) .
$$

Multiply by " $\theta$ ", to get

$$
m^{\prime}=\left(-\sqrt{3} m_{2}, \sqrt{3} m_{1},-\sqrt{3} m_{4}, \sqrt{3} m_{3},-\sqrt{3} m_{6}, \sqrt{3} m_{5}\right) .
$$

Then $c^{(i)}=m^{\prime}+a^{(i)}$ is the $i$ th candidate.

The final answer is the candidate which minimizes $N\left(w-c^{(i)}\right)$.

We used this algorithm in the Monte Carlo procedure described in $\S 2$, in order 
to estimate $G\left(E_{6}^{*}\right)$. With $5 \times 10^{6}$ random points we obtained

$$
\hat{G}\left(E_{6}^{*}\right)=0.074239 \pm 0.000018 \text {. }
$$

(As a check, $10^{7}$ points for $E_{6}$ gave $\hat{G}\left(E_{6}\right)=0.074342 \pm 0.000013$, while the exact value from [10] is $G\left(E_{6}\right)=3^{-1 / 6} 5 / 56=0.07434671 \cdots$, well within the range of the estimate.)

4. The lattices $\boldsymbol{E}_{7}$ and $\boldsymbol{E}_{7}^{*} . E_{7}$ may be obtained by applying Construction $\boldsymbol{A}$ of [27] to the little $[7,3,4]$ Hamming code $\mathscr{H}_{1}$, and a generator matrix for $E_{7}$ may be found on [37, p. 335] (see also [4], [16], [13], [27]). Similarly $E_{7}^{*}$ may be obtained from the $[7,4,3]$ Hamming code $\mathscr{H}_{2}$. Both $E_{7}$ and $E_{7}^{*}$ contain a sublattice $S_{7}=2 \mathbb{Z}^{7}$, of index 8 and 16 respectively; the coset representatives are the codewords of either $\mathscr{H}_{1}$ or $\mathscr{H}_{2}$. Since there is a trivial quantizer for $\mathbb{Z}^{7}$ (see $[11, \S$ III]), this leads to fast quantizing algorithms for $E_{7}$ and $E_{7}^{*}$; we omit the details. (Alternative algorithms, based on the sublattice $A_{7}$, were proposed in [11, $\S$ VII].) Our Monte Carlo estimate for $G\left(E_{7}^{*}\right)$, based on $10^{7}$ points, is

$$
\hat{G}\left(E_{7}^{*}\right)=0.073124 \pm 0.000013 \text {. }
$$

This is slightly better than the value for $E_{7}$, which is $0.07323063 \cdots$.

5. The Coxeter-Todd lattice $K_{12}$. The real 12-dimensional lattice $K_{12}$ was first described in [18]. It is the subject of our earlier paper [15], and further properties will be found in $\S 9$ below and in [13], [14], [27]. Since it is the densest sphere packing known in 12 dimensions [13], [27], and is also equivalent to its dual, according to the conjecture mentioned in $\S 1$ it is a good candidate for a quantizer. The same remark applies to the lattices $\Lambda_{16}$ and $\Lambda_{24}$ studied in the following sections.

Regarded as a complex 6-dimensional $\mathbb{Z}[\omega]$-lattice, $K_{12}$ has the generator matrix

$$
\left[\begin{array}{llllll}
2 & 0 & 0 & 0 & 0 & 0 \\
0 & 2 & 0 & 0 & 0 & 0 \\
0 & 0 & 2 & 0 & 0 & 0 \\
0 & 1 & 0 & 1 & \omega & \bar{\omega} \\
1 & 0 & 1 & 0 & \omega & \bar{\omega} \\
\omega & \bar{\omega} & 0 & 1 & 0 & 1
\end{array}\right],
$$

and so as a real 12-dimensional lattice it has the generator matrix shown in Fig. 4. $K_{12}$ has a sublattice isomorphic to $A_{2}^{6}$, of index 64 , namely the real version of the lattice $2 \mathbb{Z}[\omega]^{6}$. The coset representatives correspond to the codewords of the hexacode (the code over $G F(4)=\{0,1, \omega, \bar{\omega}\}$ spanned by the last three rows of (11)). This leads to a quantizing algorithm similar to those for $E_{6}$ and $E_{6}^{*}$ described in $\S 3$.

There are alternative definitions of $K_{12}$ (see [15]), which make four other sublattices visible, namely the real lattices corresponding to $\mathbb{Z}[\omega] \otimes A_{6}, \mathbb{Z}[\omega] \otimes D_{6}, \mathbb{Z}[\omega] \otimes E_{6}$, and the lattice $\left\{\left(x_{1}, \cdots, x_{6}\right):\right.$ all $\left.x_{i} \in \mathbb{Z}[\omega], \Sigma x_{i} \equiv 0(\bmod \theta)\right\}$. Each of these leads to a decoding algorithm. However the one described above seems to be the simplest.

Our Monte Carlo estimate, based on $10^{6}$ points, is

$$
\hat{G}\left(K_{12}\right)=0.070100 \pm 0.000024 \text {. }
$$

6. The Barnes-Wall lattice $\Lambda_{16}$. The lattice $\Lambda_{16}$ was first described in [3]. Other references are [13] and [27], and a generator matrix is given on page 336 of [37]. $\Lambda_{16}$ has a sublattice $2 D_{16}$ of index 32 , with coset representatives which are the codewords of the $[16,5,8]$ first-order Reed-Muller code. Then the quantizing algorithm for $D_{n}$ 


\begin{tabular}{|c|c|c|c|c|c|c|c|c|c|c|c|}
\hline 2 & 0 & 0 & 0 & 0 & 0 & 0 & 0 & 0 & 0 & 0 & 0 \\
\hline 0 & 0 & 2 & 0 & 0 & 0 & 0 & 0 & 0 & 0 & 0 & 0 \\
\hline 0 & 0 & 0 & 0 & 2 & 0 & 0 & 0 & 0 & 0 & 0 & 0 \\
\hline 0 & 0 & 1 & 0 & 0 & 0 & 1 & 0 & $-1 / 2$ & $\sqrt{3} / 2$ & $-1 / 2$ & $-\sqrt{3} / 2$ \\
\hline 1 & 0 & 0 & 0 & 1 & 0 & 0 & 0 & $-1 / 2$ & $\sqrt{3} / 2$ & $-1 / 2$ & $-\sqrt{3} / 2$ \\
\hline$-1 / 2$ & $\sqrt{3} / 2$ & $-1 / 2$ & $-\sqrt{3} / 2$ & 0 & 0 & 1 & 0 & 0 & 0 & 1 & 0 \\
\hline-1 & $\sqrt{3}$ & 0 & 0 & 0 & 0 & 0 & 0 & 0 & 0 & 0 & 0 \\
\hline 0 & 0 & -1 & $\sqrt{3}$ & 0 & 0 & 0 & 0 & 0 & 0 & 0 & 0 \\
\hline 0 & 0 & 0 & 0 & -1 & $\sqrt{3}$ & 0 & 0 & 0 & 0 & 0 & 0 \\
\hline 0 & $\underline{0}$ & $-1 / 2$ & $\sqrt{3} / 2$ & 0 & 0 & $-1 / 2$ & $\sqrt{3} / 2$ & $-1 / 2$ & $-\sqrt{3} / 2$ & 1 & 0 \\
\hline$-1 / 2$ & $\sqrt{3 / 2}$ & 0 & 0 & $-1 / 2$ & $\sqrt{3 / 2}$ & 0 & 0 & $-1 / 2$ & $-\sqrt{3 / 2}$ & 1 & 0 \\
\hline$-1 / 2$ & $-\sqrt{3 / 2}$ & 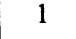 & 0 & 0 & 0 & $-1 / 2$ & $\sqrt{3 / 2}$ & 0 & 0 & $-1 / 2$ & $\sqrt{3 / 2}$ \\
\hline
\end{tabular}

FIG. 4. Generator matrix for Coxeter-Todd lattice $K_{12}$.

described in $\left[11, \S\right.$ IV] leads to an efficient quantizer for $\Lambda_{16}$. Using $2 \cdot 10^{6}$ points we found

$$
\hat{G}\left(\Lambda_{16}\right)=0.068299 \pm 0.000027 \text {. }
$$

7. The Leech lattice $\Lambda_{24}$. The Leech lattice $\Lambda_{24}$ ([5]-[8], [12]-[14], [26], [27], [36], [37]) may be constructed in many ways. The standard MOG (or miracle octad generator [7], [8], [19]) basis is shown in Fig. 5. $\Lambda_{24}$ has a sublattice $4 D_{24}$ of index 8,192 , with coset representatives

$$
2 c \text { and } 2 c+u,
$$

where $u=(-3,1,1, \cdots, 1)$, and $c$ runs through the vectors of the $[24,12,8]$ Golay code with generator matrix shown in Fig. 6 . Because of the large index of the sublattice, this is by far the slowest of our quantizing algorithms. Using 25,000 points we found

$$
\hat{G}\left(\Lambda_{24}\right)=0.065771 \pm 0.000074 \text {. }
$$

8. The number of faces of the Voronoi region. The Voronoi region $\mathscr{P}$ of a lattice $L_{n} \subseteq \mathbb{R}^{n}$ may be expressed as

$$
\mathscr{P}=\bigcap_{v \in L_{n}, v \neq 0} \mathscr{P}(v),
$$

where $\mathscr{S}(v)$ is the half-space $\left\{x \in \mathbb{R}^{n}: x \cdot v \leqq \frac{1}{2} v \cdot v\right\}$, bounded by the hyperplane

$$
\Pi(v)=\left\{x \in \mathbb{R}^{n}: x \cdot v=\frac{1}{2} v \cdot v\right\} .
$$

Of course only finitely many of the $\mathscr{S}(v)$ are really needed to define $\mathscr{P}$. Let

$$
\mathscr{P}=\bigcap_{v \in \mathscr{R}} \mathscr{P}(v)
$$

where $\mathscr{R}$ is a minimal subset of $L_{n} \mid\{0\}$ that will define $\mathscr{P}$. We call the lattice vectors in $\mathscr{R}$ relevant, and the remaining vectors of $L_{n}$ irrelevant. The number of relevant vectors is the number of $(n-1)$-dimensional faces of $\mathscr{P}$. For example, in the bodycentred cubic lattice (Fig. 1) there are 14 relevant vectors, namely the six minimal vectors and the eight vectors of the next smallest norm. 


\begin{tabular}{|c|c|c|c|c|c|}
\hline 8000 & $\begin{array}{lllll}0 & 0 & 0 & 0\end{array}$ & $\begin{array}{llllllll}0 & 0 & 0 & 0\end{array}$ & $\begin{array}{lllll}0 & 0 & 0 & 0\end{array}$ & 00000 & $\begin{array}{llll}0 & 0 & 0 & 0\end{array}$ \\
\hline 4400 & $\begin{array}{lllll}0 & 0 & 0 & 0\end{array}$ & $\begin{array}{lllll}0 & 0 & 0 & 0\end{array}$ & $\begin{array}{llll}0 & 0 & 0 & 0\end{array}$ & 00000 & 00000 \\
\hline 4040 & $\begin{array}{lllllllll}0 & 0 & 0 & 0\end{array}$ & $\begin{array}{lllllllll}0 & 0 & 0 & 0\end{array}$ & $\begin{array}{lllll}0 & 0 & 0 & 0\end{array}$ & 00000 & $\begin{array}{llll}0 & 0 & 0 & 0\end{array}$ \\
\hline 4004 & 0000 & $\begin{array}{llllllll}0 & 0 & 0 & 0\end{array}$ & $\begin{array}{llll}0 & 0 & 0 & 0\end{array}$ & $\begin{array}{llllll}0 & 0 & 0 & 0\end{array}$ & $\begin{array}{llll}0 & 0 & 0 & 0\end{array}$ \\
\hline 4000 & 00 & $\begin{array}{llllllll}0 & 0 & 0 & 0\end{array}$ & $\begin{array}{lllllll}0 & 0 & 0 & 0\end{array}$ & 0 & $\begin{array}{llll}0 & 0 & 0 & 0\end{array}$ \\
\hline 40 & & $0 \mathrm{c}$ & & & 0000 \\
\hline 4000 & 040 & $\begin{array}{llll}0 & 0 & 0 & 0\end{array}$ & 0000 & $\begin{array}{llll}0 & 0 & 0 & 0\end{array}$ & 0000 \\
\hline 2222 & 2222 & 0000 & 0000 & $\begin{array}{lllll}0 & 0 & 0 & 0\end{array}$ & 0000 \\
\hline 00 & 00 & 00 & 00 & 00000 & $\begin{array}{llll}0 & 0 & 0 & 0\end{array}$ \\
\hline 4000 & $\begin{array}{llllllll}0 & 0 & 0 & 0\end{array}$ & 0400 & 0000 & $\begin{array}{llll}0 & 0 & 0 & 0\end{array}$ & 0000 \\
\hline 4000 & $\begin{array}{llllll}0 & 0 & 0 & 0\end{array}$ & 0040 & 0000 & 0 & $\begin{array}{llll}0 & 0 & 0 & 0\end{array}$ \\
\hline 2222 & 0000 & 2222 & 0000 & $\begin{array}{llllll}0 & 0 & 0 & 0\end{array}$ & $\begin{array}{llll}0 & 0 & 0 & 0\end{array}$ \\
\hline 4000 & 00 & 00 & 00 & 00 & 000 \\
\hline 2200 & 2200 & 2200 & 2200 & $\begin{array}{lllll}0 & 0 & 0 & 0\end{array}$ & 00000 \\
\hline 2020 & 2020 & 2020 & 2020 & $\begin{array}{lllllll}0 & 0 & 0 & 0\end{array}$ & $\begin{array}{llll}0 & 0 & 0 & 0\end{array}$ \\
\hline 2002 & 2002 & 2002 & 2002 & 0000 & 0000 \\
\hline 40000 & 00 & 0 & & 40000 & 000 \\
\hline 2020 & 2002 & 2200 & $\begin{array}{llll}0 & 0 & 0 & 0\end{array}$ & 2200 & 0000 \\
\hline 2002 & 2200 & 2020 & 0000 & 2020 & 0000 \\
\hline 2200 & 2020 & 2002 & $\begin{array}{llll}0 & 0 & 0 & 0\end{array}$ & 2002 & 0000 \\
\hline 0222 & & 2000 & 20 & 00 & 2000 \\
\hline $\begin{array}{lllll}0 & 0 & 0 & 0\end{array}$ & 00000 & 2200 & 2200 & 2200 & 2200 \\
\hline $\begin{array}{lllll}0 & 0 & 0 & 0\end{array}$ & $\begin{array}{lllll}0 & 0 & 0 & 0\end{array}$ & 2020 & 2020 & 2020 & 2020 \\
\hline-3111 & 1111 & 1111 & 1111 & 1111 & 1111 \\
\hline
\end{tabular}

FIG. 5. Generator matrix for Leech lattice $\Lambda_{24}$ in standard MOG form.

\begin{tabular}{|c|c|c|c|c|c|}
\hline 11111 & 1111 & 0000 & $\begin{array}{llll}0 & 0 & 0 & 0\end{array}$ & $\begin{array}{llll}0 & 0 & 0 & 0\end{array}$ & 0000 \\
\hline $\begin{array}{lllllll}1 & 1 & 1 & 1\end{array}$ & 0000 & 11111 & $\begin{array}{llll}0 & 0 & 0 & 0\end{array}$ & 0000 & 0000 \\
\hline 11100 & 1100 & 1100 & 1100 & $\begin{array}{llll}0 & 0 & 0 & 0\end{array}$ & 0000 \\
\hline $\begin{array}{llll}1 & 0 & 1 & 0\end{array}$ & 1010 & 1010 & 1010 & 0000 & 0000 \\
\hline $\begin{array}{llll}1 & 0 & 0 & 1\end{array}$ & 1001 & 1001 & 1001 & 0000 & 0000 \\
\hline 1010 & 1001 & 1100 & 0000 & 1100 & 0000 \\
\hline $\begin{array}{llll}1 & 0 & 0 & 1\end{array}$ & 1100 & 1010 & $\begin{array}{llll}0 & 0 & 0\end{array}$ & 1010 & 0000 \\
\hline 1100 & 1010 & 1001 & $\begin{array}{llll}0 & 0 & 0\end{array}$ & 1001 & 0000 \\
\hline $\begin{array}{lllll}0 & 1 & 1 & 1\end{array}$ & 1000 & 1000 & 1000 & 1000 & 1000 \\
\hline $\begin{array}{llll}0 & 0 & 0 & 0\end{array}$ & 00000 & 1100 & 1100 & 1100 & 1100 \\
\hline 0000 & 0000 & 1010 & 1010 & 1010 & 1010 \\
\hline 1111 & 1111 & 11111 & 1111 & 1111 & 1111 \\
\hline
\end{tabular}

FIG. 6. Generator matrix for Golay code in standard MOG form.

$[10, \S \mathrm{III}]$ contains two theorems which give sufficient conditions for a lattice to have the property that the minimal vectors are the only relevant vectors. The lattices $A_{n}, D_{n}, E_{6}, E_{7}$ and $E_{8}$ have this property [10, Corollary to Theorem 5]. The following theorems can also be used to show that certain vectors are irrelevant.

THEOREM 1. If $L_{n}$ has covering radius $R_{c}$ (cf. [8]), then any vector $v \in L_{n}$ of norm $\geqq 4 R_{c}^{2}$ is irrelevant. 
Proof. Suppose $v \in L_{n}$ is relevant and $N(v) \geqq 4 R_{c}^{2}$. Then $\Pi(v)$ meets $\mathscr{P}$ in a set of positive measure. On the other hand the closest point of $\Pi(v)$ to the origin, $\frac{1}{2} v$, has norm $\geqq R_{c}^{2}$, so $\Pi(v)$ can contain at most a single vertex of $\mathscr{P}$, which is a contradiction. Q.E.D.

For example, Theorem 1 and the main result of [8] imply that for the Leech lattice only the minimal vectors and those of the next smallest norm are relevant. Thus the Voronoi region has $16,969,680$ faces.

When attempting to show that a vector $v \in L_{n}$ is irrelevant, it is sometimes possible to prove that the point $\frac{1}{2} v$ is not in the Voronoi region. In general this is not enough to prove that $v$ is irrelevant, since it is certainly possible for the hyperplane $\Pi(v)$ to intersect $\mathscr{P}$ in an asymmetric region not containing $\frac{1}{2} v$. The next theorem establishes a condition under which this does not happen.

THEOREM 2. Let $\mathscr{G}_{v}$ be the subgroup of the automorphism group Aut $\left(L_{n}\right)$ fixing a lattice vector $v$. Suppose $\mathscr{G}_{v}$ fixes exactly one 1-dimensional subspace, namely the 1-dimensional subspace containing $v$. Then if the hyperplane $\Pi(v)$ contains a point $x$ of the Voronoi region $\mathscr{P}$ different from $\frac{1}{2} v$, it follows that $\frac{1}{2} v \in \mathscr{P}$.

Proof. Consider the point

$$
y=\frac{1}{\left|\mathscr{G}_{v}\right|} \sum_{g \in \mathscr{S}_{v}} x^{g}
$$

Since $x \in \mathscr{P} \cap \Pi(v), x^{\mathscr{g}} \in \mathscr{P} \cap \Pi(v)$. Because $\mathscr{P}$ is convex, $y \in \mathscr{P} \cap \Pi(v)$. Clearly $y$ is fixed by $\mathscr{G}_{v}$. But the only point of $\Pi(v)$ fixed by $\mathscr{G}_{v}$ is $\frac{1}{2} v$. Therefore $y=\frac{1}{2} v$ and $\frac{1}{2} v \in \mathscr{P}$. Q.E.D.

An application of these theorems will be found in the following section.

9. The Voronoi region of $\boldsymbol{K}_{\mathbf{1 2}}$. We shall determine the number of faces of the Voronoi region $\mathscr{P}$ of $K_{12}$. We assume $K_{12}$ is scaled so that the minimal norm is 2 . It was shown in [15] that the covering radius of $K_{12}$ is $\sqrt{4 / 3}$, and that there are 20,412 vertices of $\mathscr{P}$ at this distance from the origin, all equivalent under Aut $\left(K_{12}\right)$. Unfortunately nothing is known about other vertices of $\mathscr{P}$.

$K_{12}$ is best studied via the corresponding 6-dimensional lattice $\Lambda_{6}^{\omega}$ (see [15]). The latter has several equivalent definitions, one of which may be seen in (11). In this section, however, another construction is more convenient, the so-called 3-base. In this form $\Lambda_{6}^{\omega}$ is defined as the set of vectors

$$
\left\{\left(x_{1}, \cdots, x_{6}\right)_{3}: \text { all } x_{i} \in \mathbb{Z}[\omega], x_{1} \equiv \cdots \equiv x_{6}(\bmod \theta) \text { and } \Sigma x_{i} \equiv 0(\bmod 3)\right\},
$$

where $\left(x_{1}, \cdots, x_{6}\right)_{3}$ is an abbreviation for $\theta^{-1}\left(x_{1}, \cdots, x_{6}\right)$.

THEOREM 3. The Voronoi region $\mathscr{P}$ of $K_{12}$ has 4,788 11 -dimensional faces, and is bounded by the hyperplanes determined by the 756 vectors of norm 2 and the 4,032 vectors of norm 3.

Proof. We shall show that the vectors of norm $\geqq 4$ in $K_{12}$ are irrelevant. Theorem 1 already implies that the vectors of norm $\geqq 6$ are irrelevant. Also it is easy to verify that Aut $\left(K_{12}\right)$ is transitive on the vectors of norms 2, 3, 4 and 5. The vectors of norms 2, 3 and 4, expressed in the 3-base, are listed in Table II of [15].

(i) The vectors of norm 5 are irrelevant. In view of the transitivity just mentioned, it is enough to show that a single norm 5 vector, say $v_{5}=(2 \theta, \theta, 0,0,0,0)_{3}$, is irrelevant. We first show that $\frac{1}{2} v_{5} \notin \mathscr{P}$. In fact it is easily checked that $\frac{1}{2} v_{5}$ is outside the hyperplane $\Pi\left(v_{2}\right)$ determined by $v_{2}=(\omega, \omega, \omega, \omega, \bar{\omega}, 1)_{3}$, and therefore by $(15)$ is not in $\mathscr{P}$. 
Next, let $\mathscr{F}$ be the stabilizer of $v_{5}$ in Aut $\left(\Lambda_{6}^{\omega}\right)$. We show that $\mathscr{F}$ only fixes a (complex) 1-dimensional subspace. Since $\mathscr{F}$ contains the diagonal matrices

$$
\operatorname{diag}\{1,1,1,1, \omega, \bar{\omega}\},
$$

where the $\omega$ and $\bar{\omega}$ may be in any of the last four positions, any vector fixed by $\mathscr{F}$ must have the form $f=(\alpha, \beta, 0,0,0,0)_{3}$. Also $\mathscr{F}$ contains the reflection

$$
R_{w}: x \mapsto x-x \cdot \bar{w} w,
$$

where $w=(1,-2,1,1,1,1)_{3}$, and so $f=(2 \beta, \beta, 0,0,0,0)_{3}$; in other words $f$ is a multiple of $v_{5}$.

Let $\mathscr{G}_{v_{5}}$ be the stabilizer in Aut $\left(K_{12}\right)$ of the real vector corresponding to $v_{5}$. Since Aut $\left(K_{12}\right)$ contains a transformation which corresponds to complex conjugation in $\Lambda_{6}^{\omega}$, only real multiples of $v_{5}$ are fixed by $\mathscr{G}_{v_{5}}$. Thus $\mathscr{G}_{v_{5}}$ only fixes a single 1 -dimensional space. We can conclude from Theorem 2 that $v_{5}$ is irrelevant.

(ii) The vectors of norm 4 are irrelevant. It is enough to consider one vector of norm 4 , say $v_{4}=(1+3 \omega, 1,1,1,1,1)_{3}$. One can now show that the equation to the hyperplane $\Pi\left(v_{4}\right)$ is already implied by the equations to the ten hyperplanes $\Pi\left(v_{2}\right)$ passing through $\frac{1}{2} v_{4}$, and defined by the following vectors $v_{2}$ :

$$
\begin{gathered}
(\omega, \bar{\omega}, 1,1,1,1)_{3}, \\
\cdots \\
(\omega, 1,1,1,1, \bar{\omega})_{3}, \\
(\theta,-\omega \theta, 0,0,0,0)_{3}, \\
\cdots \\
(\theta, 0,0,0,0,-\omega \theta)_{3} .
\end{gathered}
$$

We leave this verification to the reader, as well as the easy justification that the vectors of norms 2 and 3 are relevant. Q.E.D.

Acknowledgments. We should like to thank W. M. Kantor for some helpful discussions about $K_{12}$, and C. L. Mallows for valuable statistical advice concerning the design of the Monte Carlo experiments. These experiments were carried out on the Bell Labs Cray-1 computer, and the total computing time used (excluding test runs) was about $8 \frac{1}{2}$ hours.

\section{REFERENCES}

[1] R. P. BAmbah And N. J. A. SloAne, On a problem of Ryskov concerning lattice coverings, Acta Arithmetica, 42 (1982), pp. 107-109.

[2] E. S. BARnes AND N. J. A. SloAne, The optimal lattice quantizer in three dimensions, this Journal, 4 (1983), pp. 30-41.

[3] E. S. BArnes ANd G. E. WAll, Some extreme forms defined in terms of Abelian groups, J. Austral. Math. Soc., 1 (1959), pp. 47-63.

[4] N. Bourbaki, Groupes et algèbres de Lie, Hermann, Paris, 1968, Chap. 4-6.

[5] J. H. Conway, A characterisation of Leech's lattice, Inventiones Math., 7 (1969), pp. 137-142.

[6] - Three lectures on exceptional groups, in Finite Simple Groups, M. B. Powell and G. Higman, eds., Academic Press, New York, 1971, pp. 215-247.

[7] — The miracle octad generator, in Topics in Group Theory and Computation, M. P. J. Curran, ed., Academic Press, New York, 1977, pp. 62-68.

[8] J. H. Conway, R. A. PARker And N. J. A. SloAne, The covering radius of the Leech lattice, Proc. Royal Soc. London, A 380 (1982), pp. 261-290.

[9] J. H. ConWAy And N. J. A. Sloane, Fast 4- and 8-dimensional quantizers and decoders, National Telecommunications Record 1981, IEEE Press, New York, 1981, Vol. 3, pp. F4.2.1 to F4.2.4. 
[10] J. H. CONWAy AND N. J. A. SloANE, Voronoi regions of lattices, second moments of polytopes, and quantization, IEEE Trans. Information Theory, IT-28 (1982), pp. 211-226.

[11] - Fast quantizing and decoding algorithms for lattice quantizers and codes, IEEE Trans. Information Theory, IT-28 (1982), pp. 227-232.

[12] - Twenty-three constructions for the Leech lattice, Proc. Royal Soc. London, A 381 (1982), pp. $275-283$.

[13] - Laminated lattices, Ann. Math. 116 (1982), pp. 593-620.

[14] - Complex and integral laminated lattices, Trans. Amer. Math. Soc., in press.

[15] - The Coxeter-Todd lattice, the Mitchell group, and related sphere packings, Math. Proc. Cambridge Phil. Soc., 93 (1983), pp. 421-440.

[16] H. S. M. CoXeter, Extreme forms, Canad. J. Math., 3 (1951), pp. 391-441.

[17] - Regular Polytopes, Dover, New York, third edition, 1973.

[18] H. S. M. Coxeter And J. A. Todd, An extreme duodenary form, Canad. J. Math., 5 (1953), pp. 384-392.

[19] R. T. Curtis, On subgroups of · 0. 1: Lattice stabilizers, J. Algebra, 27 (1973), pp. 549-573.

[20] L. FEJES TÓTH, Lagerungen in der Ebene, auf der Kugel und im Raum, Springer-Verlag, Berlin, 1953. Second edition, corrected and expanded, Springer-Verlag, 1972.

[21] The PORT Mathematical Subroutine Library, P. A. Fox, ed., Bell Labs, Murray Hill, NJ, second edition, 1977.

[22] A. GERSHO, Asymptotically optimal block quantization, IEEE Trans. Information Theory, IT-25 (1979), pp. 373-380.

[23] - On the structure of vector quantizers, IEEE Trans. Information Theory, IT-28 (1982), pp. 157-166.

[24] J. H. HAlton, A retrospective and prospective survey of the Monte Carlo method, SIAM Rev., 12 (1970), pp. 1-63.

[25] J. M. Hammersley and D. C. Handscomb, Monte Carlo Methods, John Wiley, NY, 1964.

[26] J. LEECH, Notes on sphere packings, Canad. J. Math., 19 (1967), pp. 251-267.

[27] J. LEeCH AND N. J. A. SloANE, Sphere packing and error-correcting codes, Canad. J. Math., 23 (1971), pp. 718-745.

[28] Symposium on Monte Carlo Methods, H. A. Meyer, ed., John Wiley, New York, 1956.

[29] R. G. Miller, The jackknife-a review, Biometrika, 61 (1974), pp. 1-15.

[30] D. J. NEWMAN, The hexagonal theorem, IEEE Trans. Information Theory, IT-28 (1982), pp. 137-139.

[31] H.-V. NiEMEIER, Definite quadratische Formen der Dimension 24 und Diskriminante 1, J. Number Theory, 5 (1973), pp. 142-178.

[32] M. H. QUenOuIlle, Notes on bias in estimation, Biometrika, 43 (1956), pp. 353-360.

[33] S. S. RYSKOV AND E. P. BARANOVSKII, $C$-types of $n$-dimensional lattices and 5-dimensional primitive parallelohedra (with application to the theory of coverings) (in Russian), Trudy Mat. Inst. Steklov., 137 (1976); English translation in Proc. Steklov. Inst. Math., Issue 4, 1978.

[34] Yu. A. Shreider, Method of Statistical Testing: Monte Carlo Method. Elsevier, New York, 1964.

[35] N. J. A. SloAne, Codes over GF(4) and complex lattices, J. Algebra, 52 (1978), pp. 168-181.

[36] — Self-dual codes and lattices, in Relations Between Combinatorics and Other Parts of Mathematics, Proc. Symposia in Pure Mathematics, 24, American Mathematical Society, Providence, RI, 1979, pp. 273-308.

[37] _ Tables of sphere packings and spherical codes, IEEE Trans. Information Theory, IT-27 (1981), pp. 327-338.

[38] J. W. TUKEY, Bias and confidence in not-quite large samples (Abstract), Ann. Math. Stat., 29 (1958), pp. 614.

[39] P. ZADOR, Asymptotic quantization error of continuous signals and the quantization dimension, IEEE Trans. Information Theory, IT-28 (1982), pp. 139-149. 